\title{
A Generalized Nonlinear Volterra-Fredholm Type Integral Inequality and Its Application
}

\author{
Limian Zhao, ${ }^{1}$ Shanhe $\mathrm{Wu}^{2}{ }^{2}$ and Wu-Sheng Wang ${ }^{1}$ \\ ${ }^{1}$ School of Mathematics and Statistics, Hechi University, Yizhou, Guangxi 546300, China \\ ${ }^{2}$ Department of Mathematics and Computer Science, Longyan University, Longyan, Fujian 364012, China
}

Correspondence should be addressed to Shanhe Wu; shanhewu@gmail.com

Received 13 March 2014; Accepted 16 April 2014; Published 21 May 2014

Academic Editor: Kuppalapalle Vajravelu

Copyright (c) 2014 Limian Zhao et al. This is an open access article distributed under the Creative Commons Attribution License, which permits unrestricted use, distribution, and reproduction in any medium, provided the original work is properly cited.

\begin{abstract}
We establish a new nonlinear retarded Volterra-Fredholm type integral inequality. The upper bounds of the embedded unknown functions are estimated explicitly by using the theory of inequality and analytic techniques. Moreover, an application of our result to the retarded Volterra-Fredholm integral equations for estimation is given.
\end{abstract}

\section{Introduction}

Gronwall-Bellman inequality $[1,2]$ is an important tool in the study of existence, uniqueness, boundedness, oscillation, stability, invariant manifolds, and other qualitative properties of solutions of differential equations and integral equation. A lot of its generalizations in various cases can be found from the literature (e.g., [3-7]). During the past few years, some investigators have established a lot of useful and interesting integral inequalities in order to achieve various goals; see [8$18]$ and the references cited therein.

Gronwall-Bellman inequality $[1,2]$ can be stated as follows. If $u$ and $f$ are nonnegative continuous functions on an interval $[a, b]$ satisfying

$$
u(t) \leq c+\int_{a}^{t} f(s) u(s) d s, \quad t \in[a, b],
$$

for some constant $c \geq 0$, then

$$
u(t) \leq c \exp \left(\int_{a}^{t} f(s) d s\right), \quad t \in[a, b] .
$$

In 2004, Pachpatte [9] has discussed the linear VolterraFredholm type integral inequality with retardation:

$$
\begin{aligned}
u(t) \leq & k+\int_{\alpha\left(t_{0}\right)}^{\alpha(t)} a(t, s)[f(s) u(s) \\
& \left.+\int_{\alpha\left(t_{0}\right)}^{s} c(s, \tau) u(\tau) d \tau\right] d s \\
& +\int_{\alpha\left(t_{0}\right)}^{\alpha(T)} b(t, s) u(s) d s, \quad \forall t \in I .
\end{aligned}
$$

In 2011, Abdeldaim and yakout [17] studied a new integral inequality of Gronwall-Bellman-Pachpatte type:

$$
\begin{aligned}
& u(t) \leq u_{0} \\
& +\int_{\alpha\left(t_{0}\right)}^{t} f(s) u(s) \\
& \times\left[u(s)+\int_{\alpha\left(t_{0}\right)}^{s} h(\tau)\right. \\
& \times[u(\tau) \\
& \left.\left.\quad+\int_{\alpha\left(t_{0}\right)}^{\tau} g(\xi) u(\xi) d \xi\right] d \tau\right] d s .
\end{aligned}
$$


In this paper, on the basis of $[9,17]$, we discuss a new retarded nonlinear Volterra-Fredholm type integral inequality:

$$
\begin{aligned}
& u(t) \\
& \leq k
\end{aligned}
$$$$
+\int_{\alpha\left(t_{0}\right)}^{\alpha(t)} h_{1}\left(t_{1}\right)
$$$$
\times\left[f_{1}\left(t_{1}\right) \phi_{1}\left(u\left(t_{1}\right)\right)\right.
$$$$
+\int_{\alpha\left(t_{0}\right)}^{t_{1}} h_{2}\left(t_{2}\right)
$$$$
\times\left[f_{2}\left(t_{2}\right) \phi_{2}\left(u\left(t_{2}\right)\right)+\cdots\right.
$$$$
+\int_{\alpha\left(t_{0}\right)}^{t_{n-2}} h_{n-1}\left(t_{n-1}\right)
$$$$
\times\left[f_{n-1}\left(t_{n-1}\right) \phi_{n-1}\left(u\left(t_{n-1}\right)\right)\right.
$$$$
+\int_{\alpha\left(t_{0}\right)}^{t_{n-1}} h_{n}\left(t_{n}\right) \phi_{n}
$$$$
\left.\left.\left.\times\left(u\left(t_{n}\right)\right) d t_{n}\right] d t_{n-1} \ldots\right] d t_{2}\right] d t_{1}
$$$$
+\int_{\alpha\left(t_{0}\right)}^{\alpha(T)} h_{1}\left(t_{1}\right)
$$$$
\times\left[f_{1}\left(t_{1}\right) \phi_{1}\left(u\left(t_{1}\right)\right)\right.
$$$$
+\int_{\alpha\left(t_{0}\right)}^{t_{1}} h_{2}\left(t_{2}\right)
$$$$
\times\left[f_{2}\left(t_{2}\right) \phi_{2}\left(u\left(t_{2}\right)\right)+\cdots\right.
$$$$
+\int_{\alpha\left(t_{0}\right)}^{t_{n-2}} h_{n-1}\left(t_{n-1}\right)
$$$$
\times\left[f_{n-1}\left(t_{n-1}\right) \phi_{n-1}\left(u\left(t_{n-1}\right)\right)\right.
$$$$
+\int_{\alpha\left(t_{0}\right)}^{t_{n-1}} h_{n}\left(t_{n}\right) \phi_{n}
$$$$
\left.\left.\left.\times\left(u\left(t_{n}\right)\right) d t_{n}\right] d t_{n-1} \ldots\right] d t_{2}\right] d t_{1},
$$

where $k$ is a constant. The upper bound estimation of the unknown function is given by integral inequality technique, such as change of variable, amplification method, differential and integration, inverse function, and the dialectical relationship between constants and variables. Furthermore, we apply our result to retarded nonlinear Volterra-Fredholm type equations for estimation.

\section{Main Result}

Throughout this paper, $\mathbf{R}$ denotes the set of real numbers, $\mathbf{R}_{+}=[0,+\infty), I=\left[t_{0}, T\right], C^{1}(M, S)$ denotes the class of continuously differentiable functions defined on set $M$ with range in the set $S, C(M, S)$ denotes the class of continuous functions defined on set $M$ with range in the set $S$, and $\alpha^{\prime}(t)$ denotes the derived function of a function $\alpha^{\prime}(t)$.

We give the following notations used to simplify the details of presentation.

We technically define a sequence of functions $\left\{w_{i}(u)\right\}$ by $\phi_{i}(u)$ in (5), which can be defined recursively by

$$
\begin{gathered}
w_{1}(u):=\max _{\tau \in[0, u]}\left\{\phi_{1}(\tau)\right\}, \\
w_{i+1}(u):=\max _{\tau \in[0, u]}\left\{\frac{\phi_{i+1}(\tau)}{w_{i}(\tau)}\right\} w_{i}(u), \quad i=1, \ldots, n .
\end{gathered}
$$

Obviously, for all $j>i$, the function $w_{j}(u) / w_{i}(u)$ is increasing and the sequence $\left\{w_{i}(u)\right\}$ consists of nondecreasing nonnegative functions and satisfies $w_{i}(u) \geq \phi_{i}(u), i=1, \ldots, n$. Moreover,

$$
w_{i} \propto w_{i+1}, \quad i=1,2, \ldots, n-1,
$$

as defined in [4] for comparison of monotonicity of functions, because the ratios $w_{i+1}(u) / w_{i}(u), i=1, \ldots, n-1$, are all nondecreasing.

For given constant $u_{i}>0$, we define functions

$$
\begin{gathered}
W_{1}\left(u, u_{1}\right)=\int_{u_{1}}^{u} \frac{d s}{w_{1}(s)}, \\
W_{i}\left(u, u_{i}\right)=\int_{u_{i}}^{u} \frac{w_{i-1}\left(W_{1}^{-1}\left(\cdots W_{i-1}^{-1}(s) \cdots\right)\right) d s}{w_{i}\left(W_{1}^{-1}\left(\cdots W_{i-1}^{-1}(s) \cdots\right)\right)}, \\
i=2, \ldots, n,
\end{gathered}
$$

which are strictly increasing. When there is no confusion, we simply let $W_{i}(u)$ denote $W_{i}\left(u, u_{i}\right)$ and $W_{i}^{-1}$ denote its inverse. 
We define functions $\left\{H_{i}(t)\right\}(i=1,2, \ldots, n)$ :

$$
\begin{aligned}
& H_{1}(t)=\int_{\alpha\left(t_{0}\right)}^{\alpha(t)} h_{1}\left(t_{1}\right) f_{1}\left(t_{1}\right) d t_{1}, \\
& H_{2}(t)=\int_{\alpha\left(t_{0}\right)}^{\alpha(t)} h_{1}\left(t_{1}\right)\left[\int_{\alpha\left(t_{0}\right)}^{t_{1}} h_{2}\left(t_{2}\right) f_{2}\left(t_{2}\right) d t_{2}\right] d t_{1} \text {, } \\
& \text { : } \\
& H_{n-1}(t)=\int_{\alpha\left(t_{0}\right)}^{\alpha(t)} h_{1}\left(t_{1}\right) \\
& \times\left[\int_{\alpha\left(t_{0}\right)}^{t_{1}} h_{2}\left(t_{2}\right)\right. \\
& \times\left[\cdots\left[\int_{\alpha\left(t_{0}\right)}^{t_{n-2}} h_{n-1}\left(t_{n-1}\right) f_{n-1}\left(t_{n-1}\right) d t_{n-1}\right]\right. \\
& \left.\cdots] d t_{2}\right] d t_{1}, \\
& H_{n}(t)=\int_{\alpha\left(t_{0}\right)}^{\alpha(t)} h_{1}\left(t_{1}\right) \\
& \times\left[\int_{\alpha\left(t_{0}\right)}^{t_{1}} h_{2}\left(t_{2}\right)\right. \\
& \times\left[\cdots \left[\int_{\alpha\left(t_{0}\right)}^{t_{n-2}} h_{n-1}\left(t_{n-1}\right)\right.\right. \\
& \left.\times\left[\int_{\alpha\left(t_{0}\right)}^{t_{n-1}} h_{n}\left(t_{n}\right) d t_{n}\right] d t_{n-1}\right] \\
& \left.\cdots] d t_{2}\right] d t_{1} \text {. }
\end{aligned}
$$

We define function

$$
\begin{aligned}
G(u)= & W_{n}\left\{W_{n-1}\left\{\cdots\left\{W_{2}\left\{W_{1}(2 u-k)\right\}\right\} \cdots\right\}\right\} \\
- & W_{n}\left\{W _ { n - 1 } \left\{\cdots \left\{W_{2}\left\{W_{1}(u)+H_{1}(T)\right\}\right.\right.\right. \\
& \left.\left.\left.+H_{2}(T)\right\} \cdots\right\}+H_{n-1}(T)\right\} \\
& -H_{n}(T), \quad \forall u>k .
\end{aligned}
$$

Theorem 1. Suppose that $h_{n}(t), f_{i}(t), h_{i}(t) \in C\left(I, \mathbf{R}_{+}\right),(i=$ $1, \ldots, n-1), \alpha \in C^{1}(I, I)$ is nondecreasing with $\alpha(t) \leq t$ and $\alpha\left(t_{0}\right)=t_{0}$ on $I$; all $\phi_{i}$ are continuous functions with $\phi_{i}(u)>$ $0(i=1, \ldots, n)$ for $u>0, W_{i}(+\infty)=+\infty, i=1,2, \ldots, n$.
Suppose that the function $G(u)$ is increasing and $G(u)=0$ has a solution $c$ for $u>k$. If $u(t)$ satisfies (5), then

$$
\begin{aligned}
u(t) \leq & W_{1}^{-1} \\
\times & \left\{W _ { 2 } ^ { - 1 } \left\{\cdots \left\{W _ { n } ^ { - 1 } \left\{W _ { n } \left\{W _ { n - 1 } \left\{\cdots \left\{W_{2}\left\{W_{1}(c)+H_{1}(t)\right\}\right.\right.\right.\right.\right.\right.\right. \\
& \left.\left.\left.\left.\left.\left.\left.\quad+H_{2}(t)\right\} \cdots\right\}+H_{n-1}(t)\right\}+H_{n}(t)\right\}\right\} \cdots\right\}\right\}, \quad \forall t \in I,
\end{aligned}
$$

where $W_{i}^{-1}(i=1,2, \ldots, n)$ are inverse functions of $W_{i}$, respectively.

Proof. From (5) and (6), we have

$u(t)$

$\leq k$

$$
\begin{gathered}
+\int_{\alpha\left(t_{0}\right)}^{\alpha(t)} h_{1}\left(t_{1}\right) \\
\times\left[f_{1}\left(t_{1}\right) w_{1}\left(u\left(t_{1}\right)\right)\right. \\
+\int_{\alpha\left(t_{0}\right)}^{t_{1}} h_{2}\left(t_{2}\right) \\
\times\left[f_{2}\left(t_{2}\right) w_{2}\left(u\left(t_{2}\right)\right)+\cdots\right. \\
+\int_{\alpha\left(t_{0}\right)}^{t_{n-2}} h_{n-1}\left(t_{n-1}\right) \\
\times\left[f_{n-1}\left(t_{n-1}\right) w_{n-1}\left(u\left(t_{n-1}\right)\right)\right. \\
+\int_{\alpha\left(t_{0}\right)}^{t_{n-1}} h_{n}\left(t_{n}\right) w_{n} \\
\left.\left.\left.\times\left(u\left(t_{n}\right)\right) d t_{n}\right] d t_{n-1} \ldots\right] d t_{2}\right] d t_{1}
\end{gathered}
$$

$$
\begin{aligned}
& +\int_{\alpha\left(t_{0}\right)}^{\alpha(T)} h_{1}\left(t_{1}\right) \\
& \times\left[f_{1}\left(t_{1}\right) w_{1}\left(u\left(t_{1}\right)\right)\right. \\
& +\int_{\alpha\left(t_{0}\right)}^{t_{1}} h_{2}\left(t_{2}\right) \\
& \times\left[f_{2}\left(t_{2}\right) w_{2}\left(u\left(t_{2}\right)\right)+\cdots\right. \\
& +\int_{\alpha\left(t_{0}\right)}^{t_{n-2}} h_{n-1}\left(t_{n-1}\right) \\
& \times\left[f_{n-1}\left(t_{n-1}\right) w_{n-1}\left(u\left(t_{n-1}\right)\right)\right.
\end{aligned}
$$




$$
\begin{aligned}
& +\int_{\alpha\left(t_{0}\right)}^{t_{n-1}} h_{n}\left(t_{n}\right) w_{n} \\
& \left.\left.\left.\quad \times\left(u\left(t_{n}\right)\right) d t_{n}\right] d t_{n-1} \cdots\right] d t_{2}\right] d t_{1},
\end{aligned}
$$

for all $t \in I$. Let $z_{1}(t)$ denote the function on the right-hand side of (13), which is a positive and nondecreasing function on $I$. Then (13) is equivalent to

$$
u(t) \leq z_{1}(t), \quad \forall t \in I,
$$

$$
\begin{aligned}
& z_{1}\left(t_{0}\right) \\
& =k+\int_{\alpha\left(t_{0}\right)}^{\alpha(T)} h_{1}\left(t_{1}\right) \\
& \times\left[f_{1}\left(t_{1}\right) w_{1}\left(u\left(t_{1}\right)\right)\right. \\
& +\int_{\alpha\left(t_{0}\right)}^{t_{1}} h_{2}\left(t_{2}\right) \\
& \times\left[f_{2}\left(t_{2}\right) w_{2}\left(u\left(t_{2}\right)\right)+\cdots\right. \\
& +\int_{\alpha\left(t_{0}\right)}^{t_{n-2}} h_{n-1}\left(t_{n-1}\right) \\
& \times\left[f_{n-1}\left(t_{n-1}\right) w_{n-1}\left(u\left(t_{n-1}\right)\right)\right. \\
& +\int_{\alpha\left(t_{0}\right)}^{t_{n-1}} h_{n}\left(t_{n}\right) w_{n} \\
& \left.\left.\left.\times\left(u\left(t_{n}\right)\right) d t_{n}\right] d t_{n-1} \ldots\right] d t_{2}\right] d t_{1} .
\end{aligned}
$$

Differentiating $z_{1}(t)$ with respect to $t$, using (14), we have

$$
\begin{aligned}
& z_{1}^{\prime}(t) \\
& =\alpha^{\prime}(t) h_{1}(\alpha(t)) \\
& \times\left[f_{1}(\alpha(t)) w_{1}(u(\alpha(t)))\right. \\
& +\int_{\alpha\left(t_{0}\right)}^{\alpha(t)} h_{2}\left(t_{2}\right) \\
& \times\left[f_{2}\left(t_{2}\right) w_{2}\left(u\left(t_{2}\right)\right)+\cdots\right. \\
& +\int_{\alpha\left(t_{0}\right)}^{t_{n-2}} h_{n-1}\left(t_{n-1}\right) \\
& \times\left[f_{n-1}\left(t_{n-1}\right) w_{n-1}\left(u\left(t_{n-1}\right)\right)\right.
\end{aligned}
$$

$$
\begin{aligned}
& +\int_{\alpha\left(t_{0}\right)}^{t_{n-1}} h_{n}\left(t_{n}\right) w_{n} \\
& \left.\left.\left.\quad \times\left(u\left(t_{n}\right)\right) d t_{n}\right] d t_{n-1} \ldots\right] d t_{2}\right]
\end{aligned}
$$

$$
\leq \alpha^{\prime}(t) h_{1}(\alpha(t))
$$$$
\times\left[f_{1}(\alpha(t)) w_{1}\left(z_{1}(\alpha(t))\right)\right.
$$$$
+\int_{\alpha\left(t_{0}\right)}^{\alpha(t)} h_{2}\left(t_{2}\right)
$$$$
\times\left[f_{2}\left(t_{2}\right) w_{2}\left(z_{1}\left(t_{2}\right)\right)+\cdots\right.
$$$$
+\int_{\alpha\left(t_{0}\right)}^{t_{n-2}} h_{n-1}\left(t_{n-1}\right)
$$$$
\times\left[f_{n-1}\left(t_{n-1}\right) w_{n-1}\left(z_{1}\left(t_{n-1}\right)\right)\right.
$$$$
+\int_{\alpha\left(t_{0}\right)}^{t_{n-1}} h_{n}\left(t_{n}\right) w_{n}
$$$$
\left.\left.\left.\times\left(z_{1}\left(t_{n}\right)\right) d t_{n}\right] d t_{n-1} \cdots\right] d t_{2}\right],
$$

$\forall t \in I$,

by the monotonicity of $w_{1}$ and $z_{1}$ and the property of $\alpha$. From (16), we have

$$
\begin{aligned}
& \frac{z_{1}^{\prime}(t)}{w_{1}\left(z_{1}(t)\right)} \\
& \leq \alpha^{\prime}(t) h_{1}(\alpha(t)) \\
& \times\left[f_{1}(\alpha(t))\right. \\
& +\int_{\alpha\left(t_{0}\right)}^{\alpha(t)} h_{2}\left(t_{2}\right) \\
& \times\left[f_{2}\left(t_{2}\right) \frac{w_{2}\left(z_{1}\left(t_{2}\right)\right)}{w_{1}\left(z_{1}\left(t_{2}\right)\right)}+\cdots\right. \\
& +\int_{\alpha\left(t_{0}\right)}^{t_{n-2}} h_{n-1}\left(t_{n-1}\right) \\
& \times\left[f_{n-1}\left(t_{n-1}\right) \frac{w_{n-1}\left(z_{1}\left(t_{n-1}\right)\right)}{w_{1}\left(z_{1}\left(t_{n-1}\right)\right)}\right. \\
& +\int_{\alpha\left(t_{0}\right)}^{t_{n-1}} h_{n}\left(t_{n}\right)
\end{aligned}
$$


Journal of Applied Mathematics

5

$$
\left.\left.\left.\times \frac{w_{n}\left(z_{1}\left(t_{n}\right)\right)}{w_{1}\left(z_{1}\left(t_{n}\right)\right)} d t_{n}\right] d t_{n-1} \ldots\right] d t_{2}\right]
$$

$\forall t \in I$.

$$
\begin{aligned}
& +\int_{\alpha\left(t_{0}\right)}^{t_{n-1}} h_{n}\left(t_{n}\right) \\
& \left.\left.\left.\quad \times \frac{w_{n}\left(z_{1}\left(t_{n}\right)\right)}{w_{1}\left(z_{1}\left(t_{n}\right)\right)} d t_{n}\right] d t_{n-1} \cdots\right] d t_{2}\right] d t_{1},
\end{aligned}
$$

Integrating both sides of the above inequality from $t_{0}$ to $t$, we obtain

$$
\begin{aligned}
& W_{1}\left(z_{1}(t)\right) \\
& \leq W_{1}\left(z_{1}\left(t_{0}\right)\right) \\
& +\int_{\alpha\left(t_{0}\right)}^{\alpha(t)} h_{1}(s) f_{1}(s) d s \\
& +\int_{\alpha\left(t_{0}\right)}^{\alpha(t)} h_{1}\left(t_{1}\right) \\
& \times\left[\int_{\alpha\left(t_{0}\right)}^{t_{1}} h_{2}\left(t_{2}\right)\right. \\
& \times\left[f_{2}\left(t_{2}\right) \frac{w_{2}\left(z_{1}\left(t_{2}\right)\right)}{w_{1}\left(z_{1}\left(t_{2}\right)\right)}+\cdots\right. \\
& +\int_{\alpha\left(t_{0}\right)}^{t_{n-2}} h_{n-1}\left(t_{n-1}\right) \\
& \times\left[f_{n-1}\left(t_{n-1}\right) \frac{w_{n-1}\left(z_{1}\left(t_{n-1}\right)\right)}{w_{1}\left(z_{1}\left(t_{n-1}\right)\right)}\right. \\
& +\int_{\alpha\left(t_{0}\right)}^{t_{n-1}} h_{n}\left(t_{n}\right) \\
& \left.\left.\left.\times \frac{w_{n}\left(z_{1}\left(t_{n}\right)\right)}{w_{1}\left(z_{1}\left(t_{n}\right)\right)} d t_{n}\right] d t_{n-1} \cdots\right] d t_{2}\right] d t_{1}
\end{aligned}
$$$$
\leq W_{1}\left(z_{1}\left(t_{0}\right)\right)
$$$$
+\int_{\alpha\left(t_{0}\right)}^{\alpha\left(T_{1}\right)} h_{1}(s) f_{1}(s) d s
$$$$
+\int_{\alpha\left(t_{0}\right)}^{\alpha(t)} h_{1}\left(t_{1}\right)
$$$$
\times\left[\int_{\alpha\left(t_{0}\right)}^{t_{1}} h_{2}\left(t_{2}\right)\right.
$$$$
\times\left[f_{2}\left(t_{2}\right) \frac{w_{2}\left(z_{1}\left(t_{2}\right)\right)}{w_{1}\left(z_{1}\left(t_{2}\right)\right)}+\cdots\right.
$$$$
+\int_{\alpha\left(t_{0}\right)}^{t_{n-2}} h_{n-1}\left(t_{n-1}\right)
$$$$
\times\left[f_{n-1}\left(t_{n-1}\right) \frac{w_{n-1}\left(z_{1}\left(t_{n-1}\right)\right)}{w_{1}\left(z_{1}\left(t_{n-1}\right)\right)}\right.
$$

for $t_{0} \leq t \leq T_{1} \leq T ; T_{1}$ is chosen arbitrarily, where $W_{1}$ is defined by (8).

Let $z_{2}(t)$ denote the function on the right-hand side of (18), which is a positive and nondecreasing function on $\left[t_{0}, T_{1}\right]$. Then (18) is equivalent to

$$
\begin{gathered}
z_{1}(t) \leq W_{1}^{-1}\left(z_{2}(t)\right), \quad \forall t \in\left[t_{0}, T_{1}\right], \\
z_{2}\left(t_{0}\right)=W_{1}\left(z_{1}\left(t_{0}\right)\right)+\int_{\alpha\left(t_{0}\right)}^{\alpha\left(T_{1}\right)} h_{1}(s) f_{1}(s) d s .
\end{gathered}
$$

Differentiating $z_{2}(t)$ with respect to $t$, using (19), we have

$$
\begin{aligned}
& z_{2}^{\prime}(t) \\
& =\alpha^{\prime}(t) h_{1}(\alpha(t)) \\
& \times\left[\int_{\alpha\left(t_{0}\right)}^{\alpha(t)} h_{2}\left(t_{2}\right)\right. \\
& \times\left[f_{2}\left(t_{2}\right) \frac{w_{2}\left(z_{1}\left(t_{2}\right)\right)}{w_{1}\left(z_{1}\left(t_{2}\right)\right)}\right. \\
& +\int_{\alpha\left(t_{0}\right)}^{t_{2}} h_{3}\left(t_{3}\right) \\
& \times\left[f_{3}\left(t_{3}\right) \frac{w_{3}\left(z_{1}\left(t_{2}\right)\right)}{w_{1}\left(z_{1}\left(t_{2}\right)\right)}+\cdots\right. \\
& +\int_{\alpha\left(t_{0}\right)}^{t_{n-2}} h_{n-1}\left(t_{n-1}\right) \\
& \times\left[f_{n-1}\left(t_{n-1}\right) \frac{w_{n-1}\left(z_{1}\left(t_{n-1}\right)\right)}{w_{1}\left(z_{1}\left(t_{n-1}\right)\right)}\right. \\
& +\int_{\alpha\left(t_{0}\right)}^{t_{n-1}} h_{n}\left(t_{n}\right) \\
& \left.\left.\left.\left.\times \frac{w_{n}\left(z_{1}\left(t_{n}\right)\right)}{w_{1}\left(z_{1}\left(t_{n}\right)\right)} d t_{n}\right] d t_{n-1} \cdots\right] d t_{3}\right] d t_{2}\right] \\
& \leq \alpha^{\prime}(t) h_{1}(\alpha(t)) \\
& \times\left[\int_{\alpha\left(t_{0}\right)}^{\alpha(t)} h_{2}\left(t_{2}\right)\right. \\
& \times\left[f_{2}\left(t_{2}\right) \frac{w_{2}\left(W_{1}^{-1}\left(z_{2}\left(t_{2}\right)\right)\right)}{w_{1}\left(W_{1}^{-1}\left(z_{2}\left(t_{2}\right)\right)\right)}\right. \\
& +\int_{\alpha\left(t_{0}\right)}^{t_{2}} h_{3}\left(t_{3}\right) \\
& \times\left[f_{3}\left(t_{3}\right) \frac{w_{3}\left(W_{1}^{-1}\left(z_{2}\left(t_{3}\right)\right)\right)}{w_{1}\left(W_{1}^{-1}\left(z_{2}\left(t_{3}\right)\right)\right)}+\cdots\right. \\
& +\int_{\alpha\left(t_{0}\right)}^{t_{n-2}} h_{n-1}\left(t_{n-1}\right)
\end{aligned}
$$


6

Journal of Applied Mathematics

$$
\begin{aligned}
& \times\left[f_{n-1}\left(t_{n-1}\right) \frac{w_{n-1}\left(W_{1}^{-1}\left(z_{2}\left(t_{n-1}\right)\right)\right)}{w_{1}\left(W_{1}^{-1}\left(z_{2}\left(t_{n-1}\right)\right)\right)}\right. \\
& +\int_{\alpha\left(t_{0}\right)}^{t_{n-1}} h_{n}\left(t_{n}\right) \\
& \left.\left.\left.\left.\times \frac{w_{n}\left(W_{1}^{-1}\left(z_{2}\left(t_{n}\right)\right)\right)}{w_{1}\left(W_{1}^{-1}\left(z_{2}\left(t_{n}\right)\right)\right)} d t_{n}\right] d t_{n-1} \ldots\right] d t_{3}\right] d t_{2}\right], \\
& \forall t \in\left[t_{0}, T_{1}\right],
\end{aligned}
$$

by the monotonicity of $w_{i} / w_{1}(i=1,2, \ldots, n)$ and the property of $\alpha$. From (21), we have

$$
\begin{aligned}
& \frac{z_{2}^{\prime}(t) w_{1}\left(W_{1}^{-1}\left(z_{2}(t)\right)\right)}{w_{2}\left(W_{1}^{-1}\left(z_{2}(t)\right)\right)} \\
& \leq \alpha^{\prime}(t) h_{1}(\alpha(t)) \\
& \times \int_{\alpha\left(t_{0}\right)}^{\alpha(t)} h_{2}\left(t_{2}\right) f_{2}\left(t_{2}\right) d t_{2} \\
& +\alpha^{\prime}(t) h_{1}(\alpha(t)) \\
& \times \int_{\alpha\left(t_{0}\right)}^{\alpha(t)} h_{2}\left(t_{2}\right) \\
& \times\left[\int_{\alpha\left(t_{0}\right)}^{t_{2}} h_{3}\left(t_{3}\right)\right. \\
& \times\left[f_{3}\left(t_{3}\right) \frac{w_{3}\left(W_{1}^{-1}\left(z_{2}\left(t_{2}\right)\right)\right)}{w_{2}\left(W_{1}^{-1}\left(z_{2}\left(t_{2}\right)\right)\right)}+\cdots\right. \\
& +\int_{\alpha\left(t_{0}\right)}^{t_{n-2}} h_{n-1}\left(t_{n-1}\right) \\
& \times\left[f_{n-1}\left(t_{n-1}\right) \frac{w_{n-1}\left(W_{1}^{-1}\left(z_{2}\left(t_{n-1}\right)\right)\right)}{w_{2}\left(W_{1}^{-1}\left(z_{2}\left(t_{n-1}\right)\right)\right)}\right. \\
& +\int_{\alpha\left(t_{0}\right)}^{t_{n-1}} h_{n}\left(t_{n}\right) \\
& \left.\left.\left.\times \frac{w_{n}\left(W_{1}^{-1}\left(z_{2}\left(t_{n}\right)\right)\right)}{w_{2}\left(W_{1}^{-1}\left(z_{2}\left(t_{n}\right)\right)\right)} d t_{n}\right] d t_{n-1} \ldots\right] d t_{3}\right] d t_{2},
\end{aligned}
$$

for all $t \in\left[t_{0}, T_{1}\right]$. From (22), we have

$$
\begin{aligned}
& W_{2}\left(z_{2}(t)\right) \\
& \leq W_{2}\left(z_{2}\left(t_{0}\right)\right) \\
& +\int_{\alpha\left(t_{0}\right)}^{\alpha(t)} h_{1}\left(t_{1}\right) \\
& \quad \times\left[\int_{\alpha\left(t_{0}\right)}^{t_{1}} h_{2}\left(t_{2}\right) f_{2}\left(t_{2}\right) d t_{2}\right] d t_{1}
\end{aligned}
$$

$$
\begin{aligned}
& +\int_{\alpha\left(t_{0}\right)}^{\alpha(t)} h_{1}\left(t_{1}\right) \\
& \times\left[\int_{\alpha\left(t_{0}\right)}^{t_{1}} h_{2}\left(t_{2}\right)\right. \\
& \times\left[\int_{\alpha\left(t_{0}\right)}^{t_{2}} h_{3}\left(t_{3}\right)\right. \\
& \times\left[f_{3}\left(t_{3}\right) \frac{w_{3}\left(W_{1}^{-1}\left(z_{2}\left(t_{2}\right)\right)\right)}{w_{2}\left(W_{1}^{-1}\left(z_{2}\left(t_{2}\right)\right)\right)}+\cdots\right. \\
& +\int_{\alpha\left(t_{0}\right)}^{t_{n-2}} h_{n-1}\left(t_{n-1}\right) \\
& \times\left[f_{n-1}\left(t_{n-1}\right) \frac{w_{n-1}\left(W_{1}^{-1}\left(z_{2}\left(t_{n-1}\right)\right)\right)}{w_{2}\left(W_{1}^{-1}\left(z_{2}\left(t_{n-1}\right)\right)\right)}\right. \\
& +\int_{\alpha\left(t_{0}\right)}^{t_{n-1}} h_{n}\left(t_{n}\right) \\
& \left.\times \frac{w_{n}\left(W_{1}^{-1}\left(z_{2}\left(t_{n}\right)\right)\right)}{w_{2}\left(W_{1}^{-1}\left(z_{2}\left(t_{n}\right)\right)\right)} d t_{n}\right] d t_{n-1} \\
& \ldots \\
& \times
\end{aligned}
$$

$$
\begin{aligned}
& \leq W_{2}\left(z_{2}\left(t_{0}\right)\right) \\
& +\int_{\alpha\left(t_{0}\right)}^{\alpha\left(T_{1}\right)} h_{1}\left(t_{1}\right) \\
& \times\left[\int_{\alpha\left(t_{0}\right)}^{t_{1}} h_{2}\left(t_{2}\right) f_{2}\left(t_{2}\right) d t_{2}\right] d t_{1} \\
& +\int_{\alpha\left(t_{0}\right)}^{\alpha(t)} h_{1}\left(t_{1}\right) \\
& \times\left[\int_{\alpha\left(t_{0}\right)}^{t_{1}} h_{2}\left(t_{2}\right)\right. \\
& \times\left[\int_{\alpha\left(t_{0}\right)}^{t_{2}} h_{3}\left(t_{3}\right)\right. \\
& \times\left[f_{3}\left(t_{3}\right) \frac{w_{3}\left(W_{1}^{-1}\left(z_{2}\left(t_{2}\right)\right)\right)}{w_{2}\left(W_{1}^{-1}\left(z_{2}\left(t_{2}\right)\right)\right)}+\cdots\right. \\
& +\int_{\alpha\left(t_{0}\right)}^{t_{n-2}} h_{n-1}\left(t_{n-1}\right) \\
& \times\left[f_{n-1}\left(t_{n-1}\right) \frac{w_{n-1}\left(W_{1}^{-1}\left(z_{2}\left(t_{n-1}\right)\right)\right)}{w_{2}\left(W_{1}^{-1}\left(z_{2}\left(t_{n-1}\right)\right)\right)}\right. \\
& +\int_{\alpha\left(t_{0}\right)}^{t_{n-1}} h_{n}\left(t_{n}\right) \\
& \left.\times \frac{w_{n}\left(W_{1}^{-1}\left(z_{2}\left(t_{n}\right)\right)\right)}{w_{2}\left(W_{1}^{-1}\left(z_{2}\left(t_{n}\right)\right)\right)} d t_{n}\right]
\end{aligned}
$$


for all $t \in\left[t_{0}, T_{1}\right]$, where $W_{2}$ is defined by (9). Repeating the same derivation as in (19), (23), and so on, we obtain

$$
\begin{aligned}
& W_{n-2}\left(z_{n-2}(t)\right) \\
& \leq W_{n-2}\left(z_{n-2}\left(t_{0}\right)\right) \\
& +\int_{\alpha\left(t_{0}\right)}^{\alpha(t)} h_{1}\left(t_{1}\right) \\
& \times\left[\int_{\alpha\left(t_{0}\right)}^{t_{1}} h_{2}\left(t_{2}\right)\right. \\
& \left.\cdots\left[\int_{\alpha\left(t_{0}\right)}^{t_{n-3}} h_{n-2}\left(t_{n-2}\right) f_{n-2}\left(t_{n-2}\right) d t_{n-2}\right] \cdots\right] d t_{1} \\
& +\int_{\alpha\left(t_{0}\right)}^{\alpha(t)} h_{1}\left(t_{1}\right) \\
& \times\left[\int_{\alpha\left(t_{0}\right)}^{t_{1}} h_{2}\left(t_{2}\right)\right. \\
& \times[\cdots \\
& {\left[\int_{\alpha\left(t_{0}\right)}^{t_{n-2}} h_{n-1}\left(t_{n-1}\right)\right.} \\
& \times\left[f_{n-1}\left(t_{n-1}\right)\right. \\
& \times \frac{w_{n-1}\left(W_{1}^{-1}\left(W_{2}^{-1}\left(\cdots\left(W_{n-3}^{-1}\left(z_{n-2}\left(t_{n-1}\right)\right)\right) \cdots\right)\right)\right)}{w_{n-2}\left(W_{1}^{-1}\left(W_{2}^{-1}\left(\cdots\left(W_{n-3}^{-1}\left(z_{n-2}\left(t_{n-1}\right)\right)\right) \cdots\right)\right)\right)} \\
& +\int_{\alpha\left(t_{0}\right)}^{t_{n-1}} h_{n}\left(t_{n}\right) \\
& \times \frac{w_{n}\left(W_{1}^{-1}\left(W_{2}^{-1}\left(\cdots\left(W_{n-3}^{-1}\left(z_{n-2}\left(t_{n}\right)\right)\right) \cdots\right)\right)\right)}{w_{n-2}\left(W_{1}^{-1}\left(W_{2}^{-1}\left(\cdots\left(W_{n-3}^{-1}\left(z_{n-2}\left(t_{n}\right)\right)\right) \cdots\right)\right)\right)} \\
& \left.\left.\left.\left.\times d t_{n}\right] d t_{n-1}\right] \cdots\right] d t_{2}\right] d t_{1} \\
& \leq W_{n-2}\left(z_{n-2}\left(t_{0}\right)\right) \\
& +\int_{\alpha\left(t_{0}\right)}^{\alpha\left(T_{1}\right)} h_{1}\left(t_{1}\right) \\
& \times\left[\int_{\alpha\left(t_{0}\right)}^{t_{1}} h_{2}\left(t_{2}\right)\right. \\
& \left.\cdots\left[\int_{\alpha\left(t_{0}\right)}^{t_{n-3}} h_{n-2}\left(t_{n-2}\right) f_{n-2}\left(t_{n-2}\right) d t_{n-2}\right] \cdots\right] d t_{1} \\
& +\int_{\alpha\left(t_{0}\right)}^{\alpha(t)} h_{1}\left(t_{1}\right)
\end{aligned}
$$

$$
\begin{aligned}
& \times\left[\int_{\alpha\left(t_{0}\right)}^{t_{1}} h_{2}\left(t_{2}\right)\right. \\
& \times[\cdots \\
& {\left[\int_{\alpha\left(t_{0}\right)}^{t_{n-2}} h_{n-1}\left(t_{n-1}\right)\right.} \\
& \times\left[f_{n-1}\left(t_{n-1}\right)\right. \\
& \times \frac{w_{n-1}\left(W_{1}^{-1}\left(W_{2}^{-1}\left(\cdots\left(W_{n-3}^{-1}\left(z_{n-2}\left(t_{n-1}\right)\right)\right) \cdots\right)\right)\right)}{w_{n-2}\left(W_{1}^{-1}\left(W_{2}^{-1}\left(\cdots\left(W_{n-3}^{-1}\left(z_{n-2}\left(t_{n-1}\right)\right)\right) \cdots\right)\right)\right)} \\
& +\int_{\alpha\left(t_{0}\right)}^{t_{n-1}} h_{n}\left(t_{n}\right) \\
& \times \frac{w_{n}\left(W_{1}^{-1}\left(W_{2}^{-1}\left(\cdots\left(W_{n-3}^{-1}\left(z_{n-2}\left(t_{n}\right)\right)\right) \cdots\right)\right)\right)}{w_{n-2}\left(W_{1}^{-1}\left(W_{2}^{-1}\left(\cdots\left(W_{n-3}^{-1}\left(z_{n-2}\left(t_{n}\right)\right)\right) \cdots\right)\right)\right)} \\
& \left.\left.\left.\left.\times d t_{n}\right] d t_{n-1}\right] \cdots\right] d t_{2}\right] d t_{1}
\end{aligned}
$$

for all $t \in\left[t_{0}, T_{1}\right]$, where $W_{n-2}$ is defined by (9).

Let $z_{n-1}(t)$ denote the function on the right-hand side of (24), which is a positive and nondecreasing function on $\left[t_{0}, T_{1}\right]$. Then (24) is equivalent to

$$
\begin{aligned}
& z_{n-2}(t) \leq W_{n-2}^{-1}\left(z_{n-1}(t)\right), \quad \forall t \in\left[t_{0}, T_{1}\right], \\
& z_{n-1}\left(t_{0}\right) \\
& =W_{n-2}\left(z_{n-2}\left(t_{0}\right)\right) \\
& +\int_{\alpha\left(t_{0}\right)}^{\alpha\left(T_{1}\right)} h_{1}\left(t_{1}\right) \\
& \quad \times\left[\int_{\alpha\left(t_{0}\right)}^{t_{1}} h_{2}\left(t_{2}\right) \cdots\right. \\
& \left.\quad \times\left[\int_{\alpha\left(t_{0}\right)}^{t_{n-3}} h_{n-2}\left(t_{n-2}\right) f_{n-2}\left(t_{n-2}\right) d t_{n-2}\right] \ldots\right] d t_{1} .
\end{aligned}
$$

Differentiating $z_{n-1}(t)$ with respect to $t$, we have

$z_{n-1}^{\prime}(t)$

$=\alpha^{\prime}(t) h_{1}(\alpha(t))$

$\times\left[\int_{\alpha\left(t_{0}\right)}^{\alpha(t)} h_{2}\left(t_{2}\right)\right.$ 


$$
\begin{aligned}
\times[ & {\left[\int_{\alpha\left(t_{0}\right)} h_{n-1}\left(t_{n-1}\right)\right.} \\
& \times\left[f_{n-1}\left(t_{n-1}\right)\right. \\
& \times \frac{w_{n-1}\left(W_{1}^{-1}\left(W_{2}^{-1}\left(\cdots\left(W_{n-3}^{-1}\left(z_{n-2}\left(t_{n-1}\right)\right)\right) \cdots\right)\right)\right)}{w_{n-2}\left(W_{1}^{-1}\left(W_{2}^{-1}\left(\cdots\left(W_{n-3}^{-1}\left(z_{n-2}\left(t_{n-1}\right)\right)\right) \cdots\right)\right)\right)} \\
+ & \int_{\alpha\left(t_{0}\right)}^{t_{n-1}} h_{n}\left(t_{n}\right) \\
& \times \frac{w_{n}\left(W_{1}^{-1}\left(W_{2}^{-1}\left(\cdots\left(W_{n-3}^{-1}\left(z_{n-2}\left(t_{n}\right)\right)\right) \cdots\right)\right)\right)}{w_{n-2}\left(W_{1}^{-1}\left(W_{2}^{-1}\left(\cdots\left(W_{n-3}^{-1}\left(z_{n-2}\left(t_{n}\right)\right)\right) \cdots\right)\right)\right)} \\
& \left.\left.\left.\left.\times d t_{n}\right] d t_{n-1}\right] \cdots\right] d t_{2}\right],
\end{aligned}
$$

for all $t \in\left[t_{0}, T_{1}\right]$. From (27), using (25), we have

$$
\begin{aligned}
& \frac{z_{n-1}^{\prime}(t) w_{n-2}\left(W_{1}^{-1}\left(W_{2}^{-1}\left(\cdots\left(W_{n-3}^{-1}\left(z_{n-2}\left(t_{n-1}\right)\right)\right) \cdots\right)\right)\right)}{w_{n-1}\left(W_{1}^{-1}\left(W_{2}^{-1}\left(\cdots\left(W_{n-3}^{-1}\left(z_{n-2}\left(t_{n-1}\right)\right)\right) \cdots\right)\right)\right)} \\
& \leq \alpha^{\prime}(t) h_{1}(\alpha(t)) \\
& \times\left[\int_{\alpha\left(t_{0}\right)}^{\alpha(t)} h_{2}\left(t_{2}\right)\right. \\
& \times[\cdots \\
& {\left[\int_{\alpha\left(t_{0}\right)}^{t_{n-2}} h_{n-1}\left(t_{n-1}\right)\right.} \\
& \times\left[f_{n-1}\left(t_{n-1}\right)\right. \\
& +\int_{\alpha\left(t_{0}\right)}^{t_{n-1}} h_{n}\left(t_{n}\right) \\
& \times \frac{w_{n}\left(W_{1}^{-1}\left(W_{2}^{-1}\left(\cdots\left(W_{n-3}^{-1}\left(z_{n-2}\left(t_{n}\right)\right)\right) \cdots\right)\right)\right)}{w_{n-1}\left(W_{1}^{-1}\left(W_{2}^{-1}\left(\cdots\left(W_{n-3}^{-1}\left(z_{n-2}\left(t_{n}\right)\right)\right) \cdots\right)\right)\right)} \\
& \left.\left.\left.\left.\times d t_{n}\right] d t_{n-1}\right] \cdots\right] d t_{2}\right]
\end{aligned}
$$

$$
\begin{aligned}
& \leq \alpha^{\prime}(t) h_{1}(\alpha(t)) \\
& \times\left[\int_{\alpha\left(t_{0}\right)}^{\alpha(t)} h_{2}\left(t_{2}\right)\right. \\
& \times[\ldots \\
& \quad\left[\int_{\alpha\left(t_{0}\right)}^{t_{n-2}} h_{n-1}\left(t_{n-1}\right)\right. \\
& \times\left[f_{n-1}\left(t_{n-1}\right)\right. \\
& \quad\left[\int_{\alpha\left(t_{0}\right)}^{t_{n-1}} h_{n}\left(t_{n}\right)\right. \\
& \quad \times \frac{w_{n}\left(W_{1}^{-1}\left(W_{2}^{-1}\left(\cdots\left(W_{n-2}^{-1}\left(z_{n-1}\left(t_{n}\right)\right)\right) \cdots\right)\right)\right)}{w_{n-1}\left(W_{1}^{-1}\left(W_{2}^{-1}\left(\cdots\left(W_{n-2}^{-1}\left(z_{n-1}\left(t_{n}\right)\right)\right) \cdots\right)\right)\right)} \\
& \left.\left.\left.\left.\quad \times d t_{n}\right] d t_{n-1}\right] \ldots\right] d t_{2}\right],
\end{aligned}
$$

for all $t \in\left[t_{0}, T_{1}\right]$, by the monotonicity of $z_{n-1}, W_{1}^{-1}, \ldots, W_{n-2}^{-1}$ and $w_{n-2} / w_{n-1}$ and the property of $\alpha$. Integrating both sides of the above inequality from $t_{0}$ to $t$, we obtain

$$
\begin{aligned}
& W_{n-1}\left(z_{n-1}(t)\right) \\
& \leq W_{n-1}\left(z_{n-1}\left(t_{0}\right)\right) \\
& +\int_{\alpha\left(t_{0}\right)}^{\alpha(t)} h_{1}\left(t_{1}\right) \\
& \times\left[\int_{\alpha\left(t_{0}\right)}^{t_{1}} h_{2}\left(t_{2}\right)\right. \\
& \quad \times\left[\ldots\left[\int_{\alpha\left(t_{0}\right)}^{t_{n-2}} h_{n-1}\left(t_{n-1}\right) f_{n-1}\left(t_{n-1}\right) d t_{n-1}\right]\right. \\
& +\int_{\alpha\left(t_{0}\right)}^{\alpha(t)} h_{1}\left(t_{1}\right) \\
& \times\left[\int_{\alpha\left(t_{0}\right)}^{t_{1}} h_{2}\left(t_{2}\right)\right. \\
& \times\left[t_{2}\right] d t_{1} \\
& \times\left[\begin{array}{c}
\ldots \\
\times
\end{array}\right]
\end{aligned}
$$




$$
\begin{aligned}
& {\left[\int_{\alpha\left(t_{0}\right)}^{t_{n-2}} h_{n-1}\left(t_{n-1}\right)\right.} \\
& \times\left[\int_{\alpha\left(t_{0}\right)}^{t_{n-1}} h_{n}\left(t_{n}\right)\right. \\
& \times \frac{w_{n}\left(W_{1}^{-1}\left(W_{2}^{-1}\left(\cdots\left(W_{n-2}^{-1}\left(z_{n-1}\left(t_{n}\right)\right)\right) \cdots\right)\right)\right)}{w_{n-1}\left(W_{1}^{-1}\left(W_{2}^{-1}\left(\cdots\left(W_{n-2}^{-1}\left(z_{n-1}\left(t_{n}\right)\right)\right) \cdots\right)\right)\right)} \\
& \left.\left.\left.\left.\times d t_{n}\right] d t_{n-1}\right] \cdots\right] d t_{2}\right] d t_{1} \\
& \leq W_{n-1}\left(z_{n-1}\left(t_{0}\right)\right) \\
& +\int_{\alpha\left(t_{0}\right)}^{\alpha\left(T_{1}\right)} h_{1}\left(t_{1}\right) \\
& \times\left[\int_{\alpha\left(t_{0}\right)}^{t_{1}} h_{2}\left(t_{2}\right)\right. \\
& \times\left[\cdots\left[\int_{\alpha\left(t_{0}\right)}^{t_{n-2}} h_{n-1}\left(t_{n-1}\right) f_{n-1}\left(t_{n-1}\right) d t_{n-1}\right]\right. \\
& \left.\cdots] d t_{2}\right] d t_{1} \\
& +\int_{\alpha\left(t_{0}\right)}^{\alpha(t)} h_{1}\left(t_{1}\right) \\
& \times\left[\int_{\alpha\left(t_{0}\right)}^{t_{1}} h_{2}\left(t_{2}\right)\right. \\
& \times[\cdots \\
& {\left[\int_{\alpha\left(t_{0}\right)}^{t_{n-2}} h_{n-1}\left(t_{n-1}\right)\right.} \\
& \times\left[\int_{\alpha\left(t_{0}\right)}^{t_{n-1}} h_{n}\left(t_{n}\right)\right. \\
& \times \frac{w_{n}\left(W_{1}^{-1}\left(W_{2}^{-1}\left(\cdots\left(W_{n-2}^{-1}\left(z_{n-1}\left(t_{n}\right)\right)\right) \cdots\right)\right)\right)}{w_{n-1}\left(W_{1}^{-1}\left(W_{2}^{-1}\left(\cdots\left(W_{n-2}^{-1}\left(z_{n-1}\left(t_{n}\right)\right)\right) \cdots\right)\right)\right)} \\
& \left.\left.\left.\left.\times d t_{n}\right] d t_{n-1}\right] \ldots\right] d t_{2}\right] d t_{1},
\end{aligned}
$$

for all $t \in\left[t_{0}, T_{1}\right]$, where $W_{n-1}$ is defined by (9). Let $z_{n}(t)$ denote the function on the right-hand side of (29), which is a positive and nondecreasing function on $\left[t_{0}, T_{1}\right]$. Then (29) is equivalent to

$$
z_{n-1}(t) \leq W_{n-1}^{-1}\left(z_{n}(t)\right), \quad \forall t \in\left[t_{0}, T_{1}\right],
$$

$$
\begin{aligned}
& z_{n}\left(t_{0}\right) \\
& =W_{n-1}\left(z_{n-1}\left(t_{0}\right)\right) \\
& +\int_{\alpha\left(t_{0}\right)}^{\alpha\left(T_{1}\right)} h_{1}\left(t_{1}\right) \\
& \times\left[\int_{\alpha\left(t_{0}\right)}^{t_{1}} h_{2}\left(t_{2}\right)\right. \\
& \times[\ldots \\
& \quad\left[\int_{\alpha\left(t_{0}\right)}^{t_{n-2}} h_{n-1}\left(t_{n-1}\right)\right. \\
& \left.\left.\left.\times f_{n-1}\left(t_{n-1}\right) d t_{n-1}\right] \ldots\right] d t_{2}\right] d t_{1} .
\end{aligned}
$$

Differentiating $z_{n}(t)$ with respect to $t$, using (30), we have

$$
\begin{aligned}
& z_{n}^{\prime}(t) \\
& =\alpha^{\prime}(t) h_{1}(\alpha(t)) \\
& \times \int_{\alpha\left(t_{0}\right)}^{\alpha(t)} h_{2}\left(t_{2}\right) \\
& \times[\cdots \\
& {\left[\int_{\alpha\left(t_{0}\right)}^{t_{n-2}} h_{n-1}\left(t_{n-1}\right)\right.} \\
& \times\left[\int_{\alpha\left(t_{0}\right)}^{t_{n-1}} h_{n}\left(t_{n}\right)\right. \\
& \times \frac{w_{n}\left(W_{1}^{-1}\left(W_{2}^{-1}\left(\cdots\left(W_{n-2}^{-1}\left(z_{n-1}\left(t_{n}\right)\right)\right) \cdots\right)\right)\right)}{w_{n-1}\left(W_{1}^{-1}\left(W_{2}^{-1}\left(\cdots\left(W_{n-2}^{-1}\left(z_{n-1}\left(t_{n}\right)\right)\right) \cdots\right)\right)\right)} \\
& \left.\left.\left.\times d t_{n}\right] d t_{n-1}\right] \ldots\right] d t_{2} \\
& \leq \alpha^{\prime}(t) h_{1}(\alpha(t)) \\
& \times \int_{\alpha\left(t_{0}\right)}^{\alpha(t)} h_{2}\left(t_{2}\right)
\end{aligned}
$$

$\times[\cdots$

$$
\left[\int_{\alpha\left(t_{0}\right)}^{t_{n-2}} h_{n-1}\left(t_{n-1}\right)\right.
$$




$$
\begin{aligned}
& \times\left[\int_{\alpha\left(t_{0}\right)}^{t_{n-1}} h_{n}\left(t_{n}\right)\right. \\
& \times \frac{w_{n}\left(W_{1}^{-1}\left(W_{2}^{-1}\left(\cdots\left(W_{n-1}^{-1}\left(z_{n}\left(t_{n}\right)\right)\right) \cdots\right)\right)\right)}{w_{n-1}\left(W_{1}^{-1}\left(W_{2}^{-1}\left(\cdots\left(W_{n-1}^{-1}\left(z_{n}\left(t_{n}\right)\right)\right) \cdots\right)\right)\right)} \\
& \left.\left.\left.\quad \times d t_{n}\right] d t_{n-1}\right] \cdots\right] d t_{2},
\end{aligned}
$$

for all $t \in\left[t_{0}, T_{1}\right]$. From (32), we have

$$
\begin{gathered}
\frac{z_{n}^{\prime}(t) w_{n-1}\left(W_{1}^{-1}\left(W_{2}^{-1}\left(\cdots\left(W_{n-1}^{-1}\left(z_{n}\left(t_{n}\right)\right)\right) \cdots\right)\right)\right)}{w_{n}\left(W_{1}^{-1}\left(W_{2}^{-1}\left(\cdots\left(W_{n-1}^{-1}\left(z_{n}\left(t_{n}\right)\right)\right) \cdots\right)\right)\right)} \\
=\alpha^{\prime}(t) h_{1}(\alpha(t)) \\
\times \int_{\alpha\left(t_{0}\right)}^{\alpha(t)} h_{2}\left(t_{2}\right) \\
\times\left[\cdots \left[\int_{\alpha\left(t_{0}\right)}^{t_{n-2}} h_{n-1}\left(t_{n-1}\right)\right.\right. \\
\left.\times\left[\int_{\alpha\left(t_{0}\right)}^{t_{n-1}} h_{n}\left(t_{n}\right) d t_{n}\right] d t_{n-1}\right] \\
\ldots] d t_{2},
\end{gathered}
$$

for all $t \in\left[t_{0}, T_{1}\right]$. Integrating both sides of the above inequality from $t_{0}$ to $t$, we obtain

$$
\begin{aligned}
& W_{n}\left(z_{n}(t)\right)-W_{n}\left(z_{n}\left(t_{0}\right)\right) \\
& \leq \int_{\alpha\left(t_{0}\right)}^{\alpha(t)} h_{1}\left(t_{1}\right) \\
& \times\left[\int_{\alpha\left(t_{0}\right)}^{t_{1}} h_{2}\left(t_{2}\right)\right. \\
& \times\left[\ldots \left[\int_{\alpha\left(t_{0}\right)}^{t_{n-2}} h_{n-1}\left(t_{n-1}\right)\right.\right. \\
& \left.\times\left[\int_{\alpha\left(t_{0}\right)}^{t_{n-1}} h_{n}\left(t_{n}\right) d t_{n}\right] d t_{n-1}\right] \\
& \left.\ldots] d t_{2}\right] d t_{1},
\end{aligned}
$$

for all $t \in\left[t_{0}, T_{1}\right]$. Substituting (20), (26), and (31) into (35), we have

$z_{1}(t)$

$\leq W_{1}^{-1}\left\{W_{2}^{-1}\right.$

$$
\begin{aligned}
& \times\left\{\ldots \left\{W _ { n } ^ { - 1 } \left\{W _ { n } \left\{W_{n-1}\right.\right.\right.\right. \\
& \times\left\{W _ { n - 2 } \left\{\ldots \left\{W _ { 2 } \left\{W_{1}\left(z_{1}\left(t_{0}\right)\right)\right.\right.\right.\right. \\
& \left.+\int_{\alpha\left(t_{0}\right)}^{\alpha\left(T_{1}\right)} h_{1}\left(t_{1}\right) f_{1}\left(t_{1}\right) d t_{1}\right\} \\
& \left.\left.+\int_{\alpha\left(t_{0}\right)}^{\alpha\left(T_{1}\right)} h_{1}\left(t_{1}\right)\left[\int_{\alpha\left(t_{0}\right)}^{t_{1}} h_{2}\left(t_{2}\right) f_{2}\left(t_{2}\right) d t_{2}\right] d t_{1}\right\} \ldots\right\} \\
& +\int_{\alpha\left(t_{0}\right)}^{\alpha\left(T_{1}\right)} h_{1}\left(t_{1}\right)\left[\int_{\alpha\left(t_{0}\right)}^{t_{1}} h_{2}\left(t_{2}\right)[\ldots\right. \\
& \\
& \left.\left.\left.\left.+\int_{\alpha\left(t_{0}\right)}^{t_{n-3}} h_{n-2}\left(t_{n-2}\right) f_{n-2}\left(t_{n-2}\right) d t_{n-2}\right] \ldots\right] d t_{2}\right] d t_{1}\right\} \\
& +\int_{\alpha\left(t_{0}\right)}^{\alpha\left(T_{1}\right)} h_{1}\left(t_{1}\right)\left[\int_{\alpha\left(t_{0}\right)}^{t_{1}} h_{2}\left(t_{2}\right)[\ldots\right. \\
& \left.\left.\left.+\left[\int_{\alpha\left(t_{0}\right)}^{t_{n-2}} h_{n-1}\left(t_{n-1}\right) f_{n-1}\left(t_{n-1}\right) d t_{n-1}\right] \ldots\right] d t_{2}\right] d t_{1}\right\} \\
& \left.\left.\left.\left.\times\left[\int_{\alpha\left(t_{0}\right)}^{t_{n-1}} h_{1}\left(t_{1}\right)\left[\int_{\alpha\left(t_{0}\right)}^{t_{1}} h_{n}\left(t_{2}\right)\left[\ldots\left[\int_{\alpha\left(t_{0}\right)}^{t_{n-2}} h_{n-1}\left(t_{n-1}\right) d t_{n}\right] d t_{n-1}\right] \ldots\right] d t_{2}\right] d t_{1}\right\}\right\} \ldots\right\}\right\},
\end{aligned}
$$

$\forall t \in\left[t_{0}, T_{1}\right]$. 
Since $T_{1}$ is chosen arbitrarily, we have

$z_{1}(t)$

$\leq W_{1}^{-1}\left\{W_{2}^{-1}\right.$

$$
\begin{aligned}
& \times\left\{\ldots \left\{W _ { n } ^ { - 1 } \left\{W _ { n } \left\{W_{n-1}\right.\right.\right.\right. \\
& \times\left\{W _ { n - 2 } \left\{\cdots \left\{W _ { 2 } \left\{W_{1}\left(z_{1}\left(t_{0}\right)\right)\right.\right.\right.\right. \\
& \left.+\int_{\alpha\left(t_{0}\right)}^{\alpha(t)} h_{1}\left(t_{1}\right) f_{1}\left(t_{1}\right) d t_{1}\right\} \\
& \left.\left.+\int_{\alpha\left(t_{0}\right)}^{\alpha(t)} h_{1}\left(t_{1}\right)\left[\int_{\alpha\left(t_{0}\right)}^{t_{1}} h_{2}\left(t_{2}\right) f_{2}\left(t_{2}\right) d t_{2}\right] d t_{1}\right\} \ldots\right\} \\
& +\int_{\alpha\left(t_{0}\right)}^{\alpha(t)} h_{1}\left(t_{1}\right)\left[\int_{\alpha\left(t_{0}\right)}^{t_{1}} h_{2}\left(t_{2}\right)[\ldots\right. \\
& \left.\left.\left.\left.\left.\left.+\left[\int_{\alpha\left(t_{0}\right)}^{t_{n-1}} h_{n}\left(t_{n}\right) d t_{n}\right] d t_{n-1}\right] \ldots\right] d t_{2}\right] d t_{1}\right\}\right\} \ldots\right\} \\
& +\int_{\alpha\left(t_{0}\right)}^{t_{n-3}} h_{1}\left(t_{1}\right)\left[\int_{\alpha\left(t_{0}\right)}^{t_{1}} h_{2}\left(t_{2}\right)[\ldots\right. \\
& \left.\left.\left.+\int_{\alpha\left(t_{0}\right)}^{\alpha(t)} h_{1}\left(t_{1}\right)\left[\int_{\alpha\left(t_{0}\right)}^{t_{1}} h_{n-2}\left(t_{n-2}\right) d t_{n-2}\right] \ldots\right] d t_{2}\right] d t_{1}\right\} \\
& \left.\left.\left.\left[\int_{\alpha\left(t_{0}\right)}^{t_{n-2}} h_{n-1}\left(t_{n-1}\right) f_{n-1}\left(t_{n-1}\right) d t_{n-1}\right] \ldots\right] d t_{2}\right] d t_{1}\right\}
\end{aligned}
$$$$
=W_{1}^{-1}\left\{W_{2}^{-1}\right.
$$

$$
\begin{aligned}
& \times\left\{\cdots \left\{W _ { n } ^ { - 1 } \left\{W _ { n } \left\{W _ { n - 1 } \left\{\cdots \left\{W_{2}\right.\right.\right.\right.\right.\right. \\
& \left.\left.\times\left\{W_{1}\left(z_{1}\left(t_{0}\right)\right)+H_{1}(t)\right\}+H_{2}(t)\right\} \cdots\right\} \\
& \left.\left.\left.\left.\left.+H_{n-1}(t)\right\}+H_{n}(t)\right\}\right\} \cdots\right\}\right\}, \quad \forall t \in\left[t_{0}, T\right] .
\end{aligned}
$$

By the definition of $z_{1}$ and (15), we have

$$
\begin{aligned}
& 2 z_{1}\left(t_{0}\right)-k \\
& =k+2 \int_{\alpha\left(t_{0}\right)}^{\alpha(T)} h_{1}\left(t_{1}\right) \\
& \times\left[f_{1}\left(t_{1}\right) w_{1}\left(u\left(t_{1}\right)\right)\right. \\
& +\int_{\alpha\left(t_{0}\right)}^{t_{1}} h_{2}\left(t_{2}\right)
\end{aligned}
$$

$$
\times\left[f_{2}\left(t_{2}\right) w_{2}\left(u\left(t_{2}\right)\right)+\cdots\right.
$$$$
+\int_{\alpha\left(t_{0}\right)}^{t_{n-2}} h_{n-1}\left(t_{n-1}\right)
$$$$
\times\left[f_{n-1}\left(t_{n-1}\right) w_{n-1}\left(u\left(t_{n-1}\right)\right)\right.
$$$$
+\int_{\alpha\left(t_{0}\right)}^{t_{n-1}} h_{n}\left(t_{n}\right)
$$$$
\left.\times w_{n}\left(u\left(t_{n}\right)\right) d t_{n}\right] d t_{n-1}
$$$$
\left.\cdots] d t_{2}\right] d t_{1}=z_{1}(T) .
$$

From (37) and (38), we have

$$
\begin{aligned}
& \begin{array}{l}
2 z_{1}\left(t_{0}\right)-k \\
\leq W_{1}^{-1}\left\{W _ { 2 } ^ { - 1 } \left\{\cdots \left\{W _ { n } ^ { - 1 } \left\{W _ { n } \left\{W _ { n - 1 } \left\{\cdots \left\{W _ { 2 } \left\{W_{1}\left(z_{1}\left(t_{0}\right)\right)\right.\right.\right.\right.\right.\right.\right.\right. \\
\left.\left.\left.\left.\left.\left.\left.\left.+H_{1}(T)\right\}+H_{2}(T)\right\} \cdots\right\}+H_{n-1}(T)\right\}+H_{n}(T)\right\}\right\} \cdots\right\}\right\}
\end{array} \\
& \text { or } \quad \\
& \qquad \begin{array}{l}
W_{n}\left\{W_{n-1}\left\{\cdots\left\{W_{2}\left\{W_{1}\left(2 z_{1}\left(t_{0}\right)-k\right)\right\}\right\} \cdots\right\}\right\} \\
\quad-W_{n}\left\{W _ { n - 1 } \left\{\cdots \left\{W_{2}\left\{W_{1}\left(z_{1}\left(t_{0}\right)\right)+H_{1}(T)\right\}\right.\right.\right. \\
\left.\left.\left.\quad+H_{2}(T)\right\} \cdots\right\}+H_{n-1}(T)\right\}
\end{array} \\
& \quad-H_{n}(T) \leq 0 . \quad
\end{aligned}
$$

By the definition of $G$, the assumption of Theorem 1, and (40), we observe that

$$
G\left(z_{1}\left(t_{0}\right)\right) \leq 0=G(c) .
$$

Since $\mathrm{H}_{2}$ is increasing, from the last inequality and (14), we have the desired estimation (12).

We define the following functions:

$$
H_{1}(t)=\int_{\alpha\left(t_{0}\right)}^{\alpha(t)} h_{1}\left(t_{1}\right) f_{1}\left(t_{1}\right) d t_{1},
$$

$$
\begin{gathered}
H_{2}(t)=\int_{\alpha\left(t_{0}\right)}^{\alpha(t)} h_{1}\left(t_{1}\right)\left[\int_{\alpha\left(t_{0}\right)}^{t_{1}} h_{2}\left(t_{2}\right) d t_{2}\right] d t_{1}, \\
E(u)=W_{2}\left\{W_{1}(2 u-k)\right\}-W_{2}\left\{W_{1}(u)+H_{1}(T)\right\}-H_{2}(T),
\end{gathered}
$$

for all $u>k$, where $W_{i}, i=1,2$ are defined by (8) and (9), respectively.

Corollary 2. Let $n=2, f_{1}(t), f_{2}(t), h_{i}(t), \phi_{i}, W_{i}, i=1,2, \alpha$ be as in Theorem 1. Suppose that the function $E(u)$ is increasing 
and $E(u)=0$ has a solution $c$ for $u>k$. If $u(t)$ satisfies (5), then

$$
u(t) \leq W_{1}^{-1}\left\{W_{2}^{-1}\left\{W_{2}\left\{W_{1}(c)+H_{1}(t)\right\}+H_{2}(t)\right\}\right\},
$$

$\forall t \in I$,

where $W_{i}^{-1}(i=1,2)$ are inverse functions of $W_{i}$, respectively.

\section{Application}

In this section, we apply our result in Theorem 1 to investigate the retarded Volterra-Fredholm integral equations:

$x(t)$

$$
\begin{gathered}
=x_{0}+\int_{t_{0}}^{t} F_{1}\left\{s, x(s-\gamma(s)), \int_{t_{0}}^{s} F_{2}[\tau, x(\tau-\gamma(\tau))] d \tau\right\} d s \\
+\int_{t_{0}}^{T} F_{1}\left\{s, x(s-\gamma(s)), \int_{t_{0}}^{s} F_{2}[\tau, x(\tau-\gamma(\tau))] d \tau\right\} d s,
\end{gathered}
$$

for $t \in I$, where $x \in C(I, \mathbf{R}), \gamma \in C^{1}(I, I)$ is nondecreasing with $t-\gamma(t) \geq t_{0}, \gamma\left(t_{0}\right)=0, \gamma^{\prime}(t)<1, F_{1} \in C\left(I \times \mathbf{R}^{2}, \mathbf{R}\right), F_{2} \in$ $C(I \times \mathbf{R}, \mathbf{R})$. Let $\beta(t)=t-\gamma(t)$; then $\beta(t) \in C^{1}(I, I), \beta(t) \leq t$. Since $\beta^{\prime}(t)=1-\gamma^{\prime}(t)>0, \beta(t)$ is an increasing and invertible function.

The following theorem gives the bound on the solution of (44).

Theorem 3. Suppose that $F_{1}, F_{2}$ in (44) satisfy the conditions

$$
\begin{gathered}
\left|F_{1}(s, x, y)\right| \leq h_{1}(s)\left[f_{1}(s) w_{1}(|x|)+|y|\right], \\
\left|F_{2}(s, x)\right| \leq h_{2}(s) w_{2}(|x|),
\end{gathered}
$$

where $f_{1}(s), h_{1}(s), h_{2}(s), w_{1}(s)$ and $w_{2}(s)$ are as in Theorem 1; let $M=\max _{t \in I}\left(1 / \beta^{\prime}\left(\beta^{-1}(t)\right)\right)<\infty$. Assume that the function

$$
H_{3}(u)=W_{2}\left[W_{1}(2 u-k)\right]
$$

$$
\begin{aligned}
& -W_{2}\left[W_{1}(u)+\int_{\beta\left(t_{0}\right)}^{\beta(T)} h_{1}(s) f_{1}(s) d s\right] \\
& -\int_{\beta\left(t_{0}\right)}^{\beta(T)} h_{1}(s)\left[\int_{\beta\left(t_{0}\right)}^{s} h_{2}(\tau) f_{2}(\tau) d \tau\right] d s
\end{aligned}
$$

is increasing and $H_{3}(t)=0$ has a solution $c$ for $u>k$. If $x(t)$ is a solution of (44), then

$$
\begin{aligned}
|x(t)| \leq W_{1}^{-1}\left\{W _ { 2 } ^ { - 1 } \left[W_{2}\right.\right. & {\left[W_{1}(c)+\int_{\beta\left(t_{0}\right)}^{\beta(t)} M h_{1}\left(\beta^{-1}(s)\right) f_{1}\left(\beta^{-1}(s)\right) d s\right] } \\
& \left.\left.+\int_{\beta\left(t_{0}\right)}^{\beta(t)} M h_{1}\left(\beta^{-1}(s)\right)\left[\int_{\beta\left(t_{0}\right)}^{s} M h_{2}\left(\beta^{-1}(\tau)\right) d \tau\right] d s\right]\right\}, \quad \forall t \in I,
\end{aligned}
$$

where $W_{1}, W_{2}, W_{1}^{-1}$, and $W_{2}^{-1}$ are as in Theorem 1 .

Proof. Using the condition (45), we have

$$
\begin{gathered}
|x(t)| \leq\left|x_{0}\right|+\int_{t_{0}}^{t} h_{1}(s)\left[f_{1}(s) w_{1}(|x(s-\gamma(s))|)\right. \\
+\int_{t_{0}}^{s} h_{2}(\tau) \\
\left.\times w_{2}(|x(\tau-\gamma(\tau))|) d \tau\right] d s \\
+\int_{t_{0}}^{T} h_{1}(s)\left[f_{1}(s) w_{1}(|x(s-\gamma(s))|)\right. \\
\left.+\int_{t_{0}}^{s} h_{2}(\tau) w_{2}(|x(\tau-\gamma(\tau))|) d \tau\right] d s \\
=\left|x_{0}\right|+\int_{t_{0}}^{t} h_{1}(s)\left[f_{1}(s) w_{1}(|x(\beta(s))|)\right.
\end{gathered}
$$

$$
\left.+\int_{t_{0}}^{s} h_{2}(\tau) w_{2}(|x(\beta(\tau))|) d \tau\right] d s
$$

$$
+\int_{t_{0}}^{T} h_{1}(s)\left[f_{1}(s) w_{1}(|x(\beta(s))|)\right.
$$$$
\left.+\int_{t_{0}}^{s} h_{2}(\tau) w_{2}(|x(\beta(\tau))|) d \tau\right] d s
$$$$
\leq\left|x_{0}\right|+\int_{t_{0}}^{t} h_{1}(s)\left[f_{1}(s) w_{1}(|x(\beta(s))|)\right.
$$$$
+\int_{\beta\left(t_{0}\right)}^{\beta(s)} M h_{2}\left(\beta^{-1}(\tau)\right)
$$$$
\left.\times w_{2}(|x(\tau)|) d \tau\right] d s
$$

$$
\begin{array}{r}
+\int_{t_{0}}^{T} h_{1}(s)\left[f_{1}(s) w_{1}(|x(\beta(s))|)\right. \\
+\int_{\beta\left(t_{0}\right)}^{\beta(s)} M h_{2}\left(\beta^{-1}(\tau)\right)
\end{array}
$$




$$
\begin{aligned}
& \left.\times w_{2}(|x(\tau)|) d \tau\right] d s \\
& \leq\left|x_{0}\right|+\int_{\beta\left(t_{0}\right)}^{\beta(t)} M h_{1}\left(\beta^{-1}(s)\right) \\
& \times\left[f_{1}\left(\beta^{-1}(s)\right) w_{1}(|x(s)|)\right. \\
& +\int_{\beta\left(t_{0}\right)}^{\mathcal{s}} M h_{2}\left(\beta^{-1}(\tau)\right) \\
& \left.\times w_{2}(|x(\tau)|) d \tau\right] d s \\
& +\int_{\beta\left(t_{0}\right)}^{\beta(T)} M h_{1}\left(\beta^{-1}(s)\right) \\
& \times\left[f_{1}\left(\beta^{-1}(s)\right) w_{1}(|x(s)|)\right. \\
& +\int_{\beta\left(t_{0}\right)}^{s} M h_{2}\left(\beta^{-1}(\tau)\right) \\
& \left.\times w_{2}(|x(\tau)|) d \tau\right] d s,
\end{aligned}
$$

for $t \in I$, where several changes of variables are made. Applying the result of Theorem 1 to the last inequality, we obtain the desired estimation (47).

\section{Conflict of Interests}

The authors declare that there is no conflict of interests regarding the publication of this paper.

\section{Acknowledgments}

The present investigation was supported, in part, by the National Natural Science Foundation of China (no. 11161018), in part, by the NSF of Guangxi Zhuang Autonomous Region (no. 2012GXNSFAA053009), and, in part, by the Natural Science Foundation of Fujian Province of China (no. 2012J01014). The authors are grateful to the anonymous referees for their careful comments and valuable suggestions on this paper.

\section{References}

[1] T. H. Gronwall, "Note on the derivatives with respect to a parameter of the solutions of a system of differential equations," Annals of Mathematics, vol. 20, no. 4, pp. 292-296, 1919.

[2] R. Bellman, "The stability of solutions of linear differential equations," Duke Mathematical Journal, vol. 10, pp. 643-647, 1943.

[3] I. Bihari, "A generalization of a lemma of Bellman and its application to uniqueness problems of differential equations,"
Acta Mathematica Academiae Scientiarum Hungaricae, vol. 7, pp. 81-94, 1956.

[4] M. Pinto, "Integral inequalities of Bihari-type and applications," Funkcialaj Ekvacioj, vol. 33, no. 3, pp. 387-403, 1990.

[5] D. S. Mitrinović, J. E. Pečarić, and A. M. Fink, Inequalities Involving Functions and Their Integrals and Derivatives, Kluwer Academic, Dodrecht, The Netherlands, 1991.

[6] D. Bainnov and P. Simeonov, Integral Inequalities and Applications, Kluwer Academic, Dodrecht, The Netherlands, 1992.

[7] B. G. Pachpatte, Inequalities for Differential and Integral Equations, Academic Press, London, UK, 1998.

[8] O. Lipovan, "A retarded Gronwall-like inequality and its applications," Journal of Mathematical Analysis and Applications, vol. 252, no. 1, pp. 389-401, 2000.

[9] B. G. Pachpatte, "Explicit bound on a retarded integral inequality," Mathematical Inequalities \& Applications, vol. 7, no. 1, pp. 7-11, 2004.

[10] W.-S. Cheung, "Some new nonlinear inequalities and applications to boundary value problems," Nonlinear Analysis: Theory, Methods \& Applications, vol. 64, no. 9, pp. 2112-2128, 2006.

[11] W.-S. Wang, "A generalized retarded Gronwall-like inequality in two variables and applications to BVP," Applied Mathematics and Computation, vol. 191, no. 1, pp. 144-154, 2007.

[12] R. P. Agarwal, C. S. Ryoo, and Y.-H. Kim, "New integral inequalities for iterated integrals with applications," Journal of Inequalities and Applications, vol. 2007, Article ID 24385, 18 pages, 2007.

[13] W.-S. Wang and C.-X. Shen, "On a generalized retarded integral inequality with two variables," Journal of Inequalities and Applications, vol. 2008, Article ID 518646, 9 pages, 2008.

[14] W.-S. Wang, Z. Li, Y. Li, and Y. Huang, "Nonlinear retarded integral inequalities with two variables and applications," Journal of Inequalities and Applications, vol. 2010, Article ID 240790, 21 pages, 2010.

[15] W.-S. Wang, R.-C. Luo, and Z. Li, "A new nonlinear retarded integral inequality and its application," Journal of Inequalities and Applications, vol. 2010, Article ID 462163, 9 pages, 2010.

[16] W.-S. Wang, "Some generalized nonlinear retarded integral inequalities with applications," Journal of Inequalities and Applications, vol. 2012, article 31, 2012.

[17] A. Abdeldaim and M. Yakout, "On some new integral inequalities of Gronwall-Bellman-Pachpatte type," Applied Mathematics and Computation, vol. 217, no. 20, pp. 7887-7899, 2011.

[18] H. Zhou, D. Huang, W.-S. Wang, and J.-X. Xu, "Some new difference inequalities and an application to discrete-time control systems," Journal of Applied Mathematics, vol. 2012, Article ID 214609, 14 pages, 2012. 


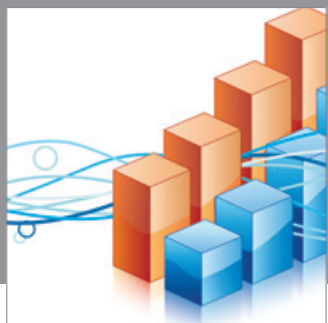

Advances in

Operations Research

mansans

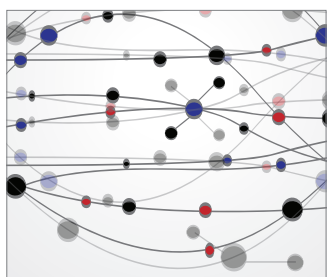

The Scientific World Journal
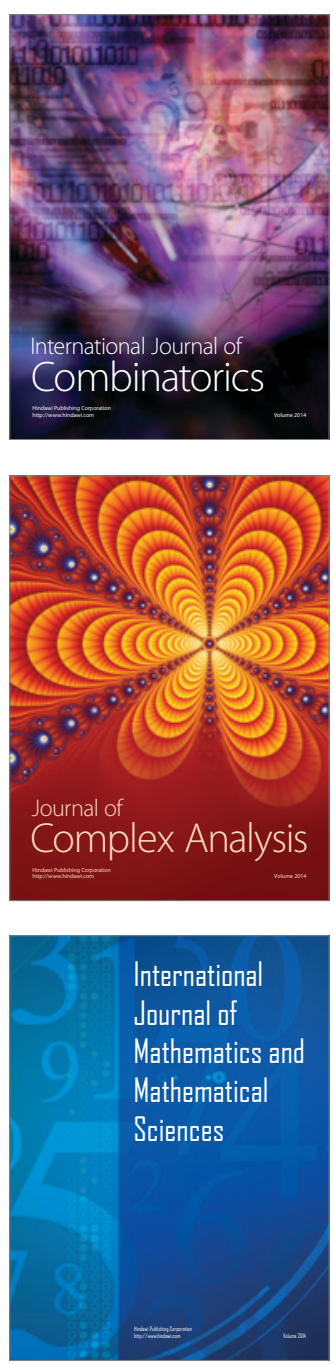
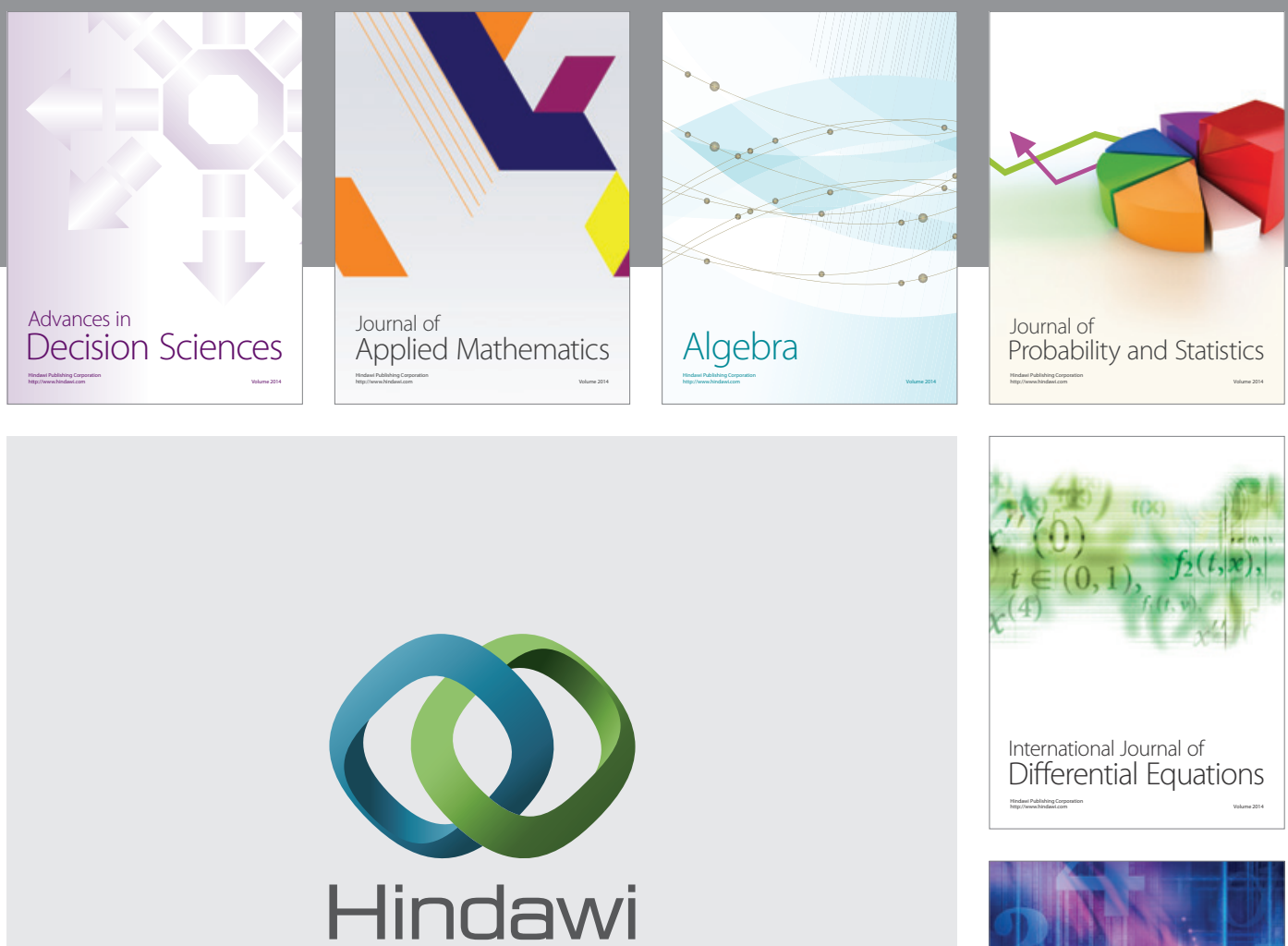

Submit your manuscripts at http://www.hindawi.com
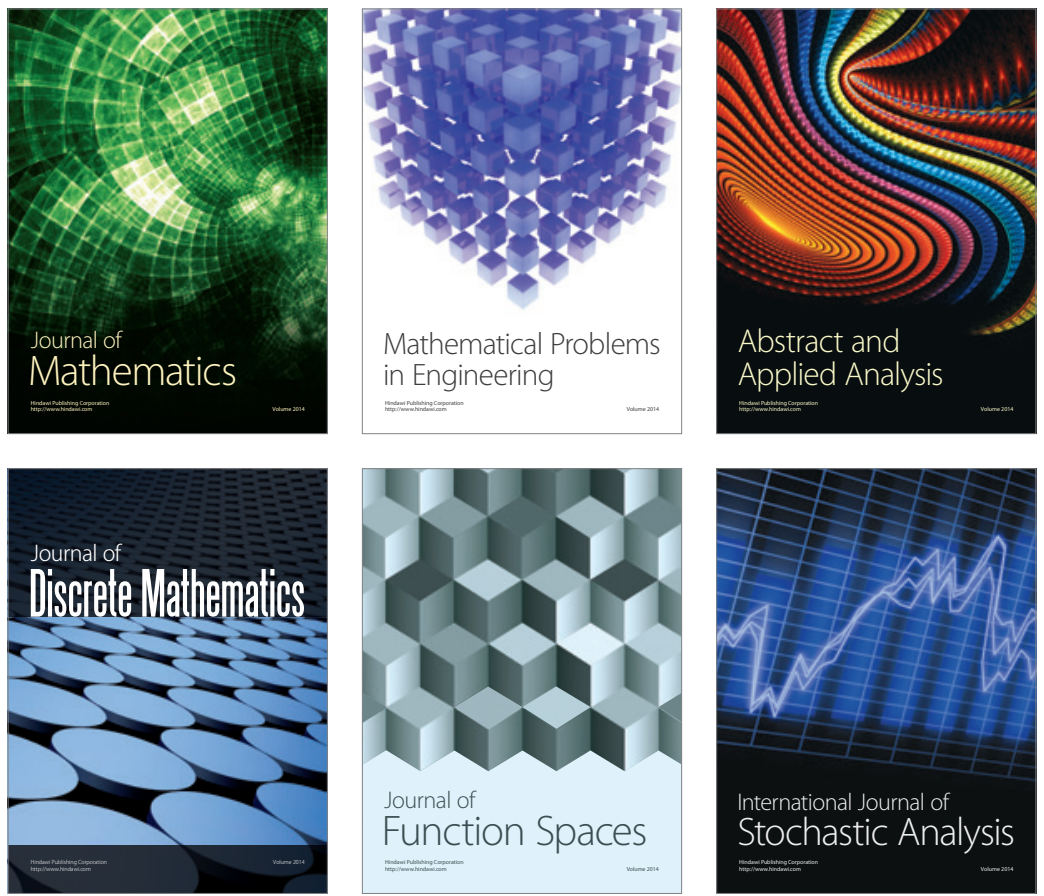

Journal of

Function Spaces

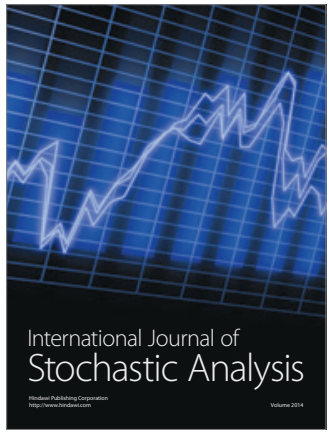

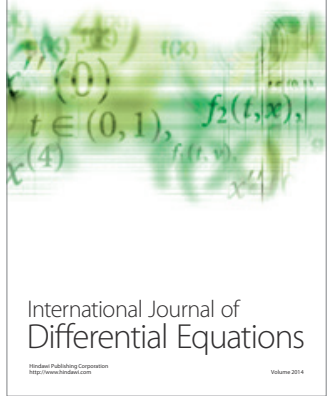
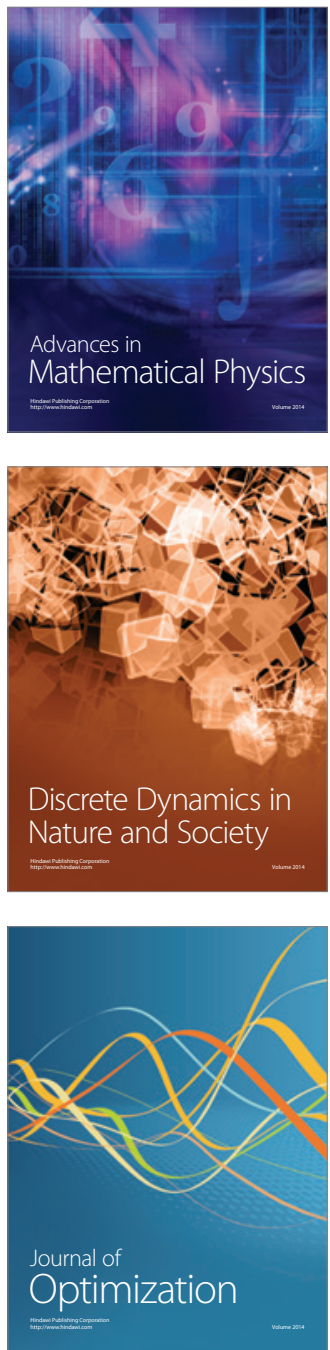